sary information to the whole of the Congo. The resolution recommended that the committee should be composed of the secretaries of the regional committees together with an appointed representative of the CPC. It was also recommended that the central committee should meet at least once annually.

One of the subjects discussed by the committees was the encouragement of original writing, preferably by Africans, and it was felt that wherever possible literature should be prepared on this basis, secondly by adaptations, and only as a last resort by translations. Other matters discussed were Mission presses, colportage work, and the desirability of increasing the circulation of the vernacular journals. Papers were also read on correspondence courses, tracts, bookshops, book clubs, planning a literature programme, and literature available and needed.

\title{
Vernacular News-Sheets in the Belgian Congo
}

THE publication of a news-sheet at Gandadjika in the Kasai marked the beginning of a movement to provide news and reading matter in the vernacular, and other areas are now following this example. The papers ate simple, generally consisting of a few stencilled pages and containing an editorial, one or more articles of general interest, an article on the history of the area, and news about sport and other events in the neighbourhood. The editorial board is composed entirely of Congolais, the chief of the territory combining his usual role with that of guide and adviser. In some territories correspondents for each native area (circonscription indigène) send in their local news. The language is generally the local vernacular, though some articles are written in French. Some news-sheets are published simultaneously in French and in the vernacular. Donations from the native area administration provide the initial finance for these publications, one of which may be started for a comparatively small sum, averaging little more than 36.000 francs. Once started, the paper depends on subscriptions and advertisements.

\section{Elisabethville University}

THE Government University at Élisabethville, Belgian Congo, was officially opened on Sunday, $1 \mathrm{r}$ November 1956, in the presence of the Minister of Colonies and professors from the four universities of Belgium. Letters of greeting were read from these as well as from the University of Lovanium (Léopoldville) whose Rector was present. The Rector of the new university outlined the reasons for its foundation and the hopes for its future; he welcomed the 42 African and European students who formed the first student body. The scholastic standard for entrance to the two Congo universities, and the level of studies pursued, will be the same as in the universities of Belgium.

\section{La sixieme Conférence des Africanistes de l'Ouest}

La sixième Conférence des Africanistes de l'Ouest s'est tenue à l'île de Sao Tomé du 21 au 28 août 1956 et a été consacrée à la fois à des séances de travail et à des excursions.

Le mérite de l'organisation revenait au Gouvernement portugais et notamment au Professeur A. Mendès Corrêa, Directeur de l'Institut supérieur des Études d'Outre-Mer de Lisbonne et membre du Comité permanent des conférences internationales des Africanistes de l'Ouest (C.I.A.O.). La session était placée sous l'égide de la Commission de Coopération technique en Afrique au sud du Sahara (C.C.T.A.) représentée à Sao Tomé par son secrétaire-général, M. Paul-Marc Henry.

Une quarantaine de congressistes, la plupart portugais, participaient à la conférence qui a été divisée en trois sections: la section physique, la section biologique et la section humaine. 
L'Institut Français d'Afrique Noire, l'Institut d'Études centrafricaines de Brazzaville, les Universités d'Ibadan et de Ghana, et l'Espagne ont été représentés.

Plus de cent communications furent présentées pendant la conférence, et en dehors des séances de travail qui se déroulèrent au lycée-collège de la ville, deux assemblées plénières se sont tenues au début et à la fin du Congrès. La séance solennelle d'inauguration a été placée sous la présidence du Gouverneur de Sao Tomé et Principe, qui a ouvert la conférence dans la salle d'honneur de l'Hôtel de Ville, et la réunion de clôture a été consacrée à l'adoption d'un certain nombre de voux concernant les problèmes scientifiques de l'Ouest africain.

Parmi les principales recommandations, il convient de noter celles se rapportant à une coopération plus étroite dans le domaine de la protection de la nature, à l'établissement d'un atlas des types de végétations de l'Afrique de l'Ouest, à une coopération permettant une étude plus poussée des plantations dites 'industrielles', à l'institution d'un comité local pour les problèmes concernant le travail et l'emploi des populations excédentaires des îles du Cap Vert, au développement de la protection et de la conservation des 'documents de l'art africain authentique', enfin à la coopération franco-portugaise pour l'étude démographique et sociologique des groupements peuls de Guinée, etc.

D'autre part, l'assemblée plénière a exprimé le vœu que la septième session puisse être organisée en 1958 en Ghana, à l'université-collège d'Achimota.

\section{Les Musées de l'IF $A N$ au Cameroun}

Le premier bâtiment qui abrita le Musée de Douala fut l'ancien siège de la Compagnie Woermann, où le Centre IFAN s'était établi depuis quelques années. En ross les collections ont été transférées dans une aile de l'Hôtel de Ville de Douala. Le hall d'entrée contient des cartes et des tableaux qui donnent quelques indications sur les milieux physique et biologique dans lesquels vivent les hommes du Cameroun. La galerie est consacrée à l'étude du milieu humain vu principalement sous l'angle de la technologie et de l'histoire. Les salles d'ethnographie présentent les divers aspects de l'art du Nord- et Sud-Cameroun et des tribus Bamum et Bamiléké.

Le Musée des Arts et Traditions Bamum de Foumban a eu pour mère la collection personnelle d'un amateur éclairé bamum, Mosé Yeyap. En 1948, après sa mort, l'IFAN transporta la collection dans un bâtiment auquel on ménagea un cachet local en adoptant la toiture de chaume des habitations bamum, et en 195s donna la formule actuelle au Musée qui devint alors le Musée des Arts et Traditions Bamum. Le Musée historique du Pays Bamum, au palais de Foumban, n'est pas un Musée de l'IFAN. Il a été créé sous le règne et sous l'impulsion du sultan Njoya, et son successeur, le sultan Seydou, en est le conservateur.

Le Musée du Diamaré (Maroua) a été créé en 1955 pour donner aux touristes quelques éléments ethnographiques afin de mieux comprendre la région qu'ils désirent visiter. En outre, il veut garder pour le savant et le chercheur certains objets des cultures autochtones, qui seront appelés fatalement à disparaître ou à dégénérer. Deux sections sont ouvertes. Elles correspondent aux grandes divisions ethniques du Diamaré : les Hamites et les Soudanais et Paléonigritiques.

L'IFAN espère ouvrir prochainement un Musée d'Art et de Folklore bamiléké à Bafoussam dont les collections sont en cours d'installation.

\section{Notes sur les migrations soudanaises en Ghana}

LES renseignements recueillis depuis plusieurs décennies au Soudan Français ont fait apparaître la fréquence de voyages effectués par un très grand nombre d'individus apparte- 